\title{
DETECCIÓN DE OUTLIERS USANDO MÉTRICAS DE DISTANCIA Y ANÁLISIS CLUSTER
}

\author{
Santiago Cartagena Agudelo \\ Ingeniería Matemática \\ Universidad EAFIT \\ scartagena@eafit.edu.co \\ Pablo Buitrago Jaramillo \\ Ingeniería Matemática \\ Universidad EAFIT \\ pbuitragoj@eafit.edu.co \\ Camilo Cossio Alzate \\ Ingeniería Matemática \\ Universidad EAFIT \\ ccossioadeafit.edu.co \\ Andrés Ospina Patiño \\ Ingeniería Matemática \\ Universidad EAFIT \\ aospinapdeafit.edu.co
}

Junio 2021 


\section{Índice}

$\begin{array}{lr}\text { 1. Resumen } & 3\end{array}$

2. Palabras claves $\quad 3$

3. Abstract $r$

$\begin{array}{lr}\text { 4. Introducción } & 5\end{array}$

5. Objetivos $\quad 6$

6. Marco teórico $r$

6.1. Distancia Euclidiana . . . . . . . . . . . . . . . . . . . 6

6.2. Distancia de Manhattan . . . . . . . . . . . . . . . . . . . . . . 6

6.3. Similitud del coseno . . . . . . . . . . . . . . . . . . 7

6.4. Distancia de Mahalanobis . . . . . . . . . . . . . . . . . . . . 8

6.5. K-means Clustering . . . . . . . . . . . . . . . . . . . . . 9

6.6. Agglomerative Nesting Clustering . . . . . . . . . . . . . . . . . . . 10

7. Implementación computacional $r$

7.1. Detección de Outliers . . . . . . . . . . . . . . . . . . . . . . . 12

7.2. Análisis Cluster . . . . . . . . . . . . . . . . . . . 15

$\begin{array}{lr}\text { 8. } \text { Resultados } & 17\end{array}$

8.1. Detección de Outliers mediante las métricas . . . . . . . . . . . . . . . . . . . 19

8.2. Análisis Cluster . . . . . . . . . . . . . . . . . . . . . . 22

$\begin{array}{lr}\text { 9. Conclusiones } & 23\end{array}$ 


\section{Resumen}

En muchas técnicas apropiadas para realizar ciencia de datos y machine learning, es necesario poder medir la separación entre diferentes registros. Por ejemplo, en los métodos de análisis de clúster es necesario para obtener un grado de similitud entre los registros. La forma de hacer esto es utilizando las distancias o métricas, asumiendo de esta forma que los datos son puntos en un espacio de n dimensiones.

La medida de distancias juega un papel importante en la agrupación de puntos de datos. Elegir la medida de distancia correcta para un conjunto de datos determinado no es un problema trivial, y requiere de ciertos conocimientos previos para llevar a cabo este proceso de buena forma. En este trabajo, se estudian y se implementan varias de las medidas de distancia más conocidas hoy en día, tales como la distancia de Mahalanobis, la distancia euclídea, la distancia de Manhattan y, la distancia del coseno, la cual llamó el interés de los autores por su nombre, a pesar de no ser tan conocida como las otras tres, dicho análisis se llevo a cabo con el objetivo de observar la aplicación de dichas distancias en la vida real con un conjunto de datos real tomado de acciones diarias de compañías desde la yahoo finance.

La implementación de las distancias, así como los análisis estadísticos realizados a su aplicación y los resultados se llevaron a cabo en el lenguaje de programación Python.

\section{Palabras claves}

Outliers, clusters, clasificación, métricas de distancia, scatterplot, activos financieros, comparación, análisis. 


\section{Abstract}

In many techniques appropriate for conducting data science and machine learning, it is necessary to be able to measure the separation between different records. For example, in cluster analysis methods it is necessary to obtain a degree of similarity between the records. The way to do this is by using distances or metrics, thus assuming that the data are points in an n-dimensional space.

Distance measurements play an important role in grouping data points. Choosing the correct distance measure for a given data set is not a trivial problem, and requires some prior knowledge to carry out this process in a good way. In this work, several of the most well-known distance measurements are studied and implemented today, such as the Mahalanobis distance, the Euclidean distance, the Manhattan distance and the cosine distance, which attracted the interest of the authors by name, despite not being as well known as the other three, said analysis was carried out with the aim of observing the application of these distances in real life with a set of real data taken from daily actions of companies from yahoo finance.

The implementation of the distances, as well as the statistical analyzes carried out to its application and the results were carried out in the Python programming language. 


\section{Introducción}

El cálculo de distancia se usa ampliamente para determinar el grado de similitud o no de dos vectores. Las medidas de distancia entre poblaciones y dentro de poblaciones, han sido utilizadas en numerosos campos científicos: antropología, agricultura, biología, genética, economía, lingüística, psicología, sociología, etc, asimismo el papel de las distancias estadísticas cuando se trata de problemas como pruebas de hipótesis, pruebas de bondad de ajuste, técnicas de clasificación, análisis de clusters, valores outliers y los métodos de detección y estimación de la densidad son de gran importancia. El uso de medidas de distancia nos permite cuantificar la cercanía entre dos objetos estadísticos. Estos objetos pueden ser dos variables aleatorias, dos distribuciones de probabilidad, funciones generadoras de momento, un punto muestral individual y distribuciones de probabilidad o dos muestras individuales. Existen muchas medidas estadísticas de distancia, en esta investigación se llevará un análisis a profundidad de cuatro de esas distancias. Además de la distancia Euclidea, distancia Manhattan, Coseno y similitud del coseno, y la distancia de Mahalanobis, esta última posee una particularidad, y es que cuenta con la ventaja de detectar valores atípicos o outliers multivariados. Los valores outliers o atípicos son aquellos datos que se desvían del comportamiento general de la mayoría de los datos. Estos valores atípicos tienen una definición diferente en los textos, por ejemplo, un valor atípico se desvía tanto de otras observaciones como para despertar sospechas de que fue generado por un mecanismo diferente, ver [5]. A menudo, determinar la métrica a utilizar representa un gran desafío, pues la medida de similitud adecuada depende de los datos que se estén analizando, y no en todos los casos se tiene un conjunto de datos o dataset completamente limpio. También, la similitud o disimilitud entre objetos se puede utilizar para detectar valores atípicos en los datos o para realizar la clasificación del vecino más cercano o k-nearest neighbor.

Por otra parte,en las aplicaciones de minería de datos, como la agrupación en clústeres, el análisis de valores atípicos y la clasificación del vecino más cercano, necesitamos formas de evaluar qué tan similares o diferentes son los objetos en comparación entre sí.

Un clúster es una colección de objetos de datos de manera que los objetos dentro de un clúster son similares entre sí y diferentes a los objetos de otros clústeres, ver [1]. El análisis de valores atípicos también emplea técnicas basadas en agrupamiento para identificar posibles valores atípicos como objetos que son muy diferentes de otros. 


\section{Objetivos}

- Comparar el desempeño de diferentes métricas en la detección de outliers.

- Analizar el desempeño de la distancia de Mahalanobis calculada de una manera más robusta mediante el uso de otro estadístico (la mediana).

- Implementar un análisis Cluster del conjunto de datos para identificar grupos de datos que difieren significativamente unos entre otros.

\section{Marco teórico}

\subsection{Distancia Euclidiana}

La distancia Euclidiana es la más común y usada entre las métricas. Esta representa la distancia más corta que hay entre los dos objetos que están siendo comparados, pues esta dada por la distancia en línea recta entre dos puntos de datos en el espacio euclidiano, y se deduce a partir del teorema de Pitágoras. Esta puede ser generalizada para dimensiones más grandes, como en el caso que se tengan las observaciones de $n$ individuos para $p$ variables, y sea el individuo $i$ representado como un punto $x_{i} \in R^{p}$ [3]. La distancia euclídea entre $i, j$ esta dada por

$$
d_{2}(i, j)=\sqrt{\sum_{k=1}^{p}\left(x_{i k}-x_{j k}\right)^{2}}
$$

\subsection{Distancia de Manhattan}

La distancia de Manhattan también se conoce como city block distance en Inglés, que es la suma de las distancias de todos los atributos. Para los dos puntos de datos $x, y$ en las dimensiones del espacio d. Si $u=\left(x_{1}, y_{1}\right)$ y $v=\left(x_{2}, y_{2}\right)$ son dos puntos, entonces la distancia Manhattan entre u y v está dada por:

$$
M H(u, v)=\left|x_{1}-x_{2}\right|+\left|y_{1}-y_{2}\right|
$$

Ahora, si en vez de dos dimensiones los puntos tienen $n$-dimensiones, por ejemplo $u=\left(x_{1}, x_{2}, x_{3}, \ldots, x_{n}\right)$ y $v=\left(y_{1}, y_{2}, y_{3}, \ldots, y_{n}\right)$, entonces la ecuación (2) puede generalizarse de la siguiente forma definiendo la distancia de Manhattan entre $u$ y $v$. 


$$
\begin{gathered}
M H(u, v)=\left|x_{1}-x_{2}\right|+\left|y_{1}-y_{2}\right|+\left|x_{3}-x_{4}\right|+\left|y_{3}-y_{4}\right|+|\ldots|+\left|x_{n}-y_{n}\right| \\
=\sum_{i=1}^{n}\left|x_{i}-y_{i}\right|
\end{gathered}
$$

Se llama distancia de Manhattan porque es la distancia que recorrería un automóvil o cualquier vehículo dentro una ciudad cualquiera, por ejemplo en Manhattan corresponde a la ruta de $p$ a $q$ a lo largo de calles horizontales y verticales al igual que las carreteras en el área de dicho barrio en Nueva York, que es uno de los 5 grandes barrios esta gran ciudad estadounidense, allí los edificios están dispuestos en bloques cuadrados y las calles rectas se cruzan en ángulos rectos. Esto explica los otros términos con los que se le conoce a esta distancia como City Block y Geometría del Taxi o cab distances.

Como sugiere [2], la distancia de Manhattan (norma L1) puede ser preferible sobre la distancia euclidiana (norma L2) para el caso de datos de alta dimensión. Por otra parte, cabe destacar que los autores de artículos acerca de la distancia Manhattan en general incluso van un paso más allá y sugieren utilizar distancias de norma Lk, con un valor fraccional de k, para datos dimensionales muy altos con el fin de mejorar los resultados de los algoritmos basados en distancias, como la agrupación en clústeres.

\subsection{Similitud del coseno}

Es importante aclarar que la similitud y la distancia están relacionadas pero no son exactamente lo mismo. Como bien lo presentan Xia, Zhang y Li en su artículo Learning similarity with cosine similarity ensemble [c2], la similitud se usa para medir las características comunes entre dos instancias y la distancia se adopta para indicar las diferencias entre ellas. Primero se puede calcular la distancia entre dos instancias y luego establecer un umbral apropiado para decidir si son similares o no. Dos instancias serán más similares a medida que disminuya la distancia entre ellas. En este caso que se tiene la similitud, es usual, obtener la distancia de la diferencia entre 1 y la similitud.

Dados dos vectores $\mathrm{N}$ dimensionales $\vec{v} \mathrm{y} \vec{w}$, la similaridad del coseno como es presentado en [7] entre ellos esta dada por

$$
\cos \theta=\frac{\vec{v} \cdot \vec{w}}{\|\vec{v}\|\|\vec{w}\|}=\frac{\sum_{i=1}^{N} v_{i} \times w_{i}}{\sqrt{\sum_{i=1}^{N} v_{i}^{2}} \sqrt{\sum_{i=1}^{N} w_{i}^{2}}}
$$

donde $\theta$ es el ángulo entre $\vec{v}$ y $\vec{w}$. Esta medida determina si dos vectores apuntan aproximadamente en la misma dirección, de esta manera, si se obtiene un valor 0 , quiere decir que los objetos no son iguales, 
mientras que, entre mayor sea el valor de similitud, mayor será la similitud entre los objetos.

La gran ventaja de esta métrica es que, a diferencia de la euclidiana, no es sensible a las magnitudes, y es por ello que existen diversas aplicaciones, principalmente en la similitud de documentos en el análisis de texto, recuperación de información, agrupamiento de documentos de texto, taxonomía biológica y mapeo de características genéticas. Sin embargo, en algunas ocasiones dos patrones con valores de atributo muy diferentes pueden tener una medida de similitud muy alta, lo cual es indeseable y por tanto se acostumbra a utilizar una métrica de similitud de coseno ajustada que solucione este problema.

\subsection{Distancia de Mahalanobis}

La distancia de Mahalanobis fue introducida por Mahalanobis en 1936, su utilida se basa en que es una forma de determinar la similitud entre dos variables aleatorias multidimensionales. Su diferencia principal con la distancia euclídea es que esta cuenta con la correlación entre las variables aleatorias. [8]

Supongamos que una población $\Omega$ está caracterizada por $p$ variables aleatorias, siendo $\mu=\left(\mu_{1}, \ldots, \mu_{p}\right)^{\prime}$ el vector de medias y $\Sigma$ la matriz de covarianzas no singular. La distancia de Mahalanobis $M(i, j)$ entre dos individuos $i, j$ representados por los vectores $x_{i}, x_{j}$ se define como [3]

$$
M^{2}(i, j)=\left(x_{i}-x_{j}\right)^{\prime} \Sigma^{-1}\left(x_{i}-x_{j}\right)
$$

Análogamente, la distancia entre un individuo $i$ y la población $\Omega$ es

$$
M^{2}(i, \Omega)=\left(x_{i}-\mu\right)^{\prime} \Sigma^{-1}\left(x_{i}-\mu\right)
$$

Y la distancia entre dos poblaciones $\Omega_{1}, \Omega_{2}$ se define como

$$
M^{2}\left(\Omega_{1}, \Omega_{2}\right)=\left(\mu_{1}-\mu_{2}\right)^{\prime} \Sigma^{-1}\left(\mu_{1}-\mu_{2}\right)
$$

Esta distancia presenta las siguientes propiedades [3]:

- $M(i, j) \geq 0$ y $M(i, j)=0$ si y sólo si $x_{i}=x_{j}$

- $M(i, j)=M(j, i)$

- $M(i, j) \leq M(i, k)+M(j, k)$

- $M(i, j)$ es invariante por cambios de escala 
- Introduciendo el cambio de variable $y=\Sigma^{-1 / 2} x$, es fácil ver que la distancia de Mahalanobis es Euclídea

- Es una distancia normalizada

- Tiene en cuenta la correlación, es decir, la redundancia entre las variables

\subsection{K-means Clustering}

La idea del clustering es encontrar grupos en un conjunto de datos, en los que los individuos pertenecientes a este tengan características similares, mientras que los que no pertenecen difieran lo más posible. Esto es de gran utilidad en el análisis de datos, pues permite una mayor especificidad en los grupos, encontrando características que los representan y que posteriormente pueden ser utilizadas para predecir la pertenencia de un nuevo individuo a uno de estos grupos.

En el agrupamiento de k-means, cada grupo está representado por su centro que corresponde a la media de puntos asignados al grupo, pretendiendo minimizar la variación total dentro de la agrupación, la cual se define como la suma de las distancias al cuadrado, de las distancias euclidianas entre los elementos y el centroide correspondiente.

El primer paso al utilizar la agrupación de k-means es indicar el número de agrupaciones (k) que se generarán en la solución final. El algoritmo comienza seleccionando aleatoriamente k objetos del conjunto de datos para que sirvan como centros iniciales para los grupos. A continuación, cada uno de los objetos restantes se asigna a su centroide más cercano, donde más cercano se define utilizando la distancia euclidiana entre el objeto y la media del grupo. Después del paso de asignación, el algoritmo calcula el nuevo valor medio de cada grupo. Ahora que se han recalculado los centros, se vuelve a comprobar cada observación para ver si podría estar más cerca de un grupo diferente. Todos los objetos se reasignan nuevamente utilizando los medios de clúster actualizados. Los pasos de actualización de centroide y asignación de conglomerados se repiten iterativamente hasta que las asignaciones de conglomerados dejan de cambiar (es decir, hasta que se logra la convergencia).

Las principales ventajas del algoritmo k-means son su simplicidad y velocidad, lo que le permite ejecutarlo en grandes conjuntos de datos. Debido a una agrupación inicial aleatoria, no produce el mismo resultado con cada ejecución del algoritmo, como bien es presentado en [6]. 


\subsection{Agglomerative Nesting Clustering}

También conocido como AGNES, es un conjunto de metodos de agrupación que funcionan de forma ascendente. Cada elemento (dato) es considerado un cluster de un elemento, en cada iteración del algoritmo las parejas de clusters más similares son agrupadas en un cluster más grande, el proceso se repite hasta que todos los elementos pertenezcan a un único cluster. En este caso se agrupan según la similitud de las distancias con respecto al centroide de los clusters. Estos métodos no requieren de un número $\mathrm{k}$ de clusters para operar el algoritmo, pero existen técnicas para determinar el número optimo de cluster según los datos.

\section{Implementación computacional}

Las Bibliotecas a importar necesarias para usar el código son:

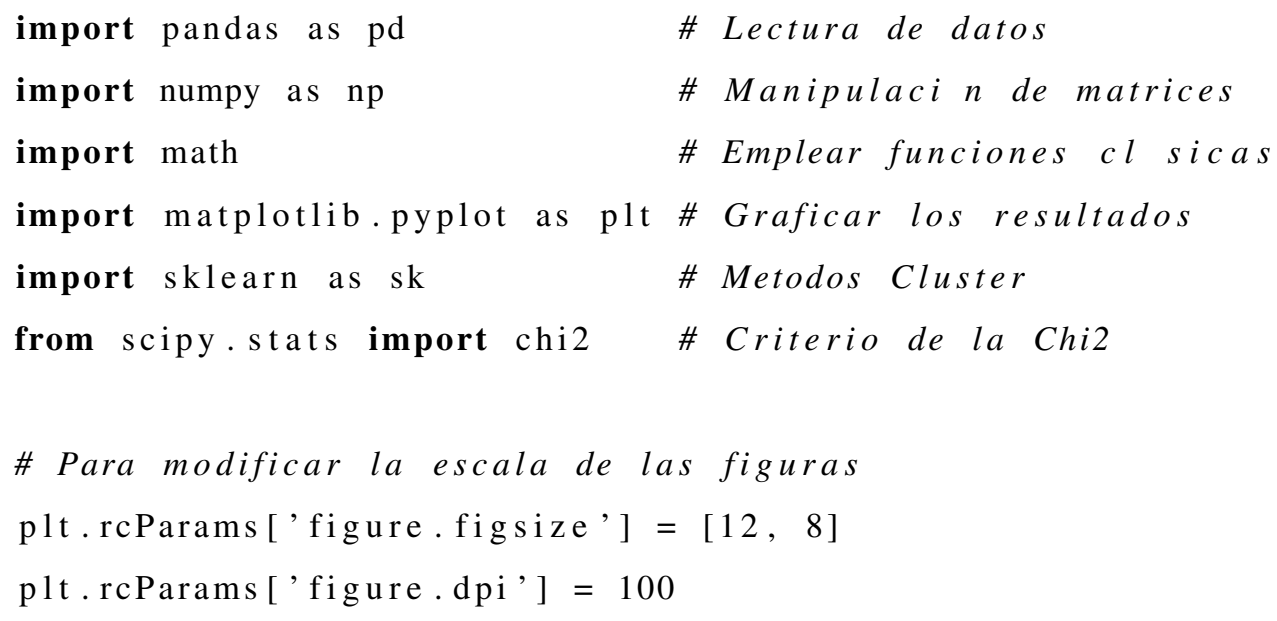

Los métodos de distancias Euclidea, del Coseno y Manhattan reciben un vector $\mathbf{X}$ y un vector $\mathbf{Y}$ como parámetros (cada uno contiene las coordenadas de un punto respectivamente) y devuelven la distancia entre ambos puntos según la métrica.

\section{Distancia Euclidea}

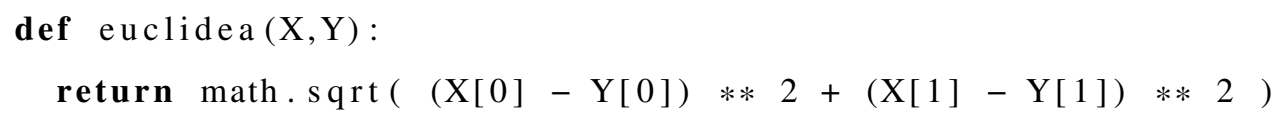

\section{Distancia del Coseno}

def $\cos (\mathrm{X}, \mathrm{Y})$ :

return $1-n p \cdot \operatorname{dot}(X, Y) /(n p .1 i n a l g \cdot \operatorname{norm}(X) * n p \cdot \operatorname{linalg} \cdot \operatorname{norm}(Y))$ 


\section{Distancia Manhattan}

def manhatt $(X, Y)$ :

return np.linalg.norm $(\mathrm{X}[0]-\mathrm{Y}[0])+\mathrm{np} .1 \mathrm{inalg} \cdot \operatorname{norm}(\mathrm{X}[1]-\mathrm{Y}[1])$

\section{Distancia Mahalanobis}

def mahalanobis $(\mathrm{X}, \mathrm{Y})$ :

vect $=[]$

means $=[n p \cdot \operatorname{mean}(\mathrm{X}), \mathrm{np} \cdot \operatorname{mean}(\mathrm{Y})]$

$\mathrm{S}=\mathrm{np} \cdot \operatorname{cov}(\mathrm{X}, \mathrm{Y})$

for $\mathrm{i}$ in range(len $(\mathrm{X}))$ :

point $=$ np.array $((\mathrm{X}[\mathrm{i}]-\operatorname{means}[0], \mathrm{Y}[\mathrm{i}]-\operatorname{means}[1]))$

dist $=n p \cdot s q r t(n p \cdot d o t(n p \cdot d o t($ point, np.linalg.inv $(S))$, np.transpose $($ point

) ) )

vect. append ( dist)

return vect

\section{Distancia Mahalanobis con respecto a la media}

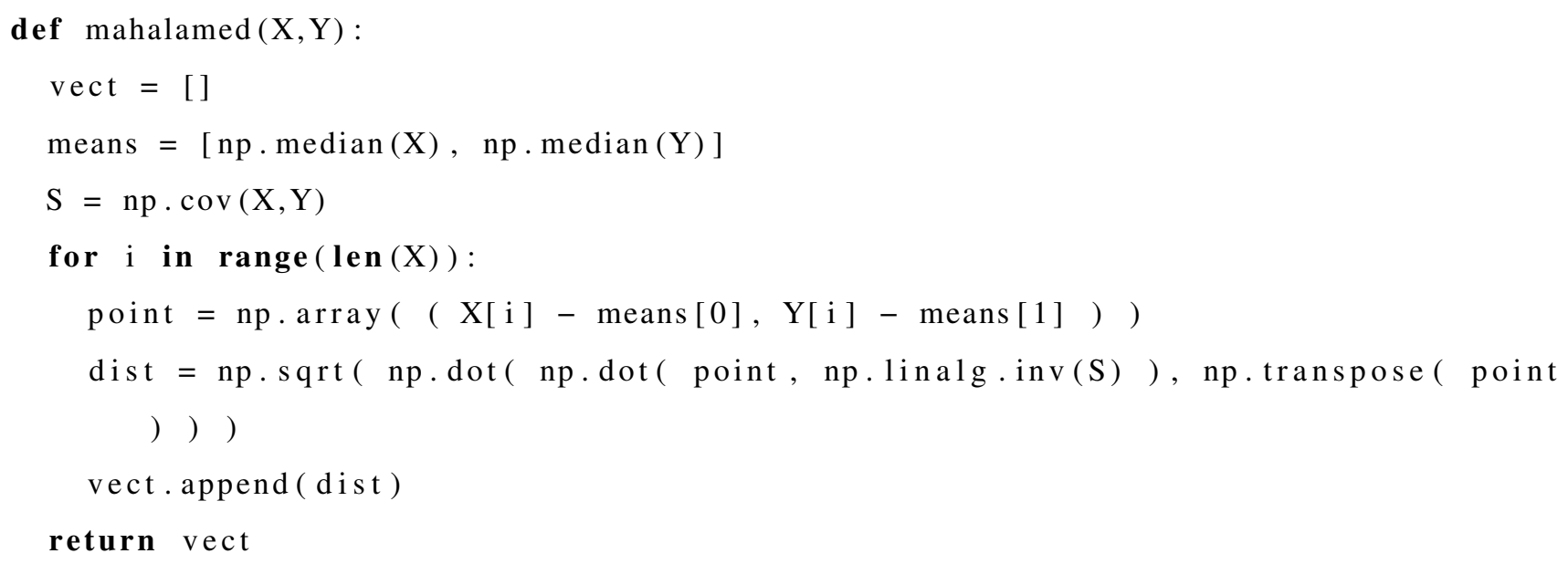

\section{Cov Mediana}

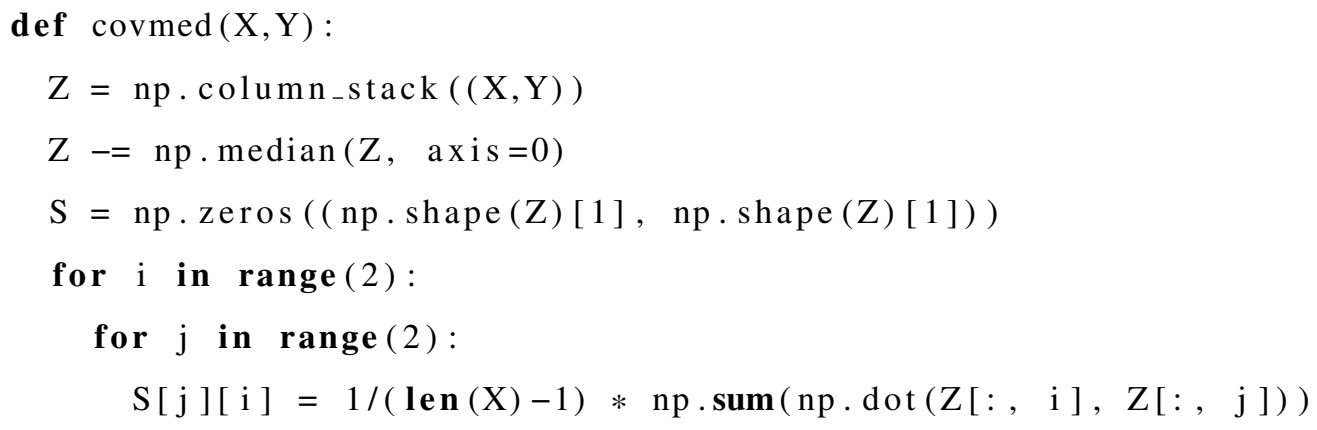




\section{Distancia Mahalanobis calculada con la CovMediana}

def mahacovmed $(X, Y)$ :

vect $=[]$

means $=[\mathrm{np} \cdot \operatorname{mean}(\mathrm{X}), \mathrm{np} \cdot \operatorname{mean}(\mathrm{Y})]$

$S=\operatorname{covmed}(X, Y)$

for $\mathrm{i}$ in range(len $(\mathrm{X}))$ :

point $=$ np.array $((\mathrm{X}[\mathrm{i}]-\operatorname{means}[0], \mathrm{Y}[\mathrm{i}]-\operatorname{means}[1]))$

dist $=n p \cdot s q r t(n p \cdot d o t(n p \cdot d o t($ point, np.linalg.inv $(S))$, np.transpose $($ point

) ) )

vect . append (dist)

return vect

\section{Criterio Chi2}

def qchisq(NumVariables):

return chi2.ppf(0.95, NumVariables)

\subsection{Detección de Outliers}

\section{Preparación de los datos}

\# Leemos los datos a emplear

APPL $=$ pd.read_csv ('AAPL.csv')

TSLA $=$ pd.read_csv ('TSLA. csv')

\# Seleccionamos la columna de interes

variable $1=$ APPL. iloc $[:, 4]$

variable $2=$ TSLA. iloc $[:, 4]$

\section{Scatter Plot Inicial}

plt. scatter (variable1, variable2)

plt.xlabel ('variable1')

plt.ylabel ('variable2')

plt.title('Scatter Plot inicial')

\section{Calculo de las distancias}

\# Creamos los vectores para guardar las diferentes distancias

$\mathrm{dist}_{-} \mathrm{eucl}=[]$

dist $_{-} \cos =[]$ 


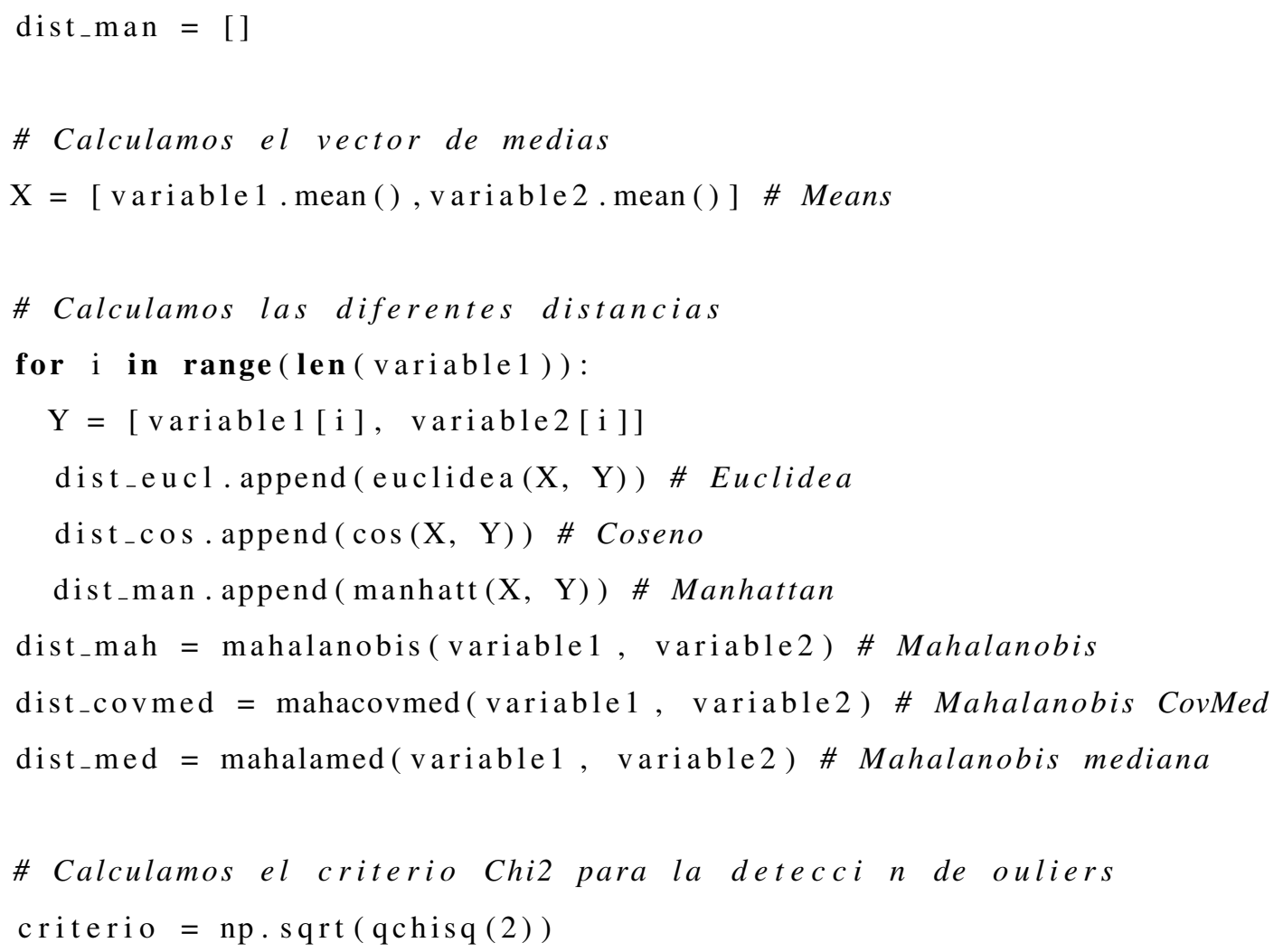

Plots de la detección de outliers con las diferentes distancias.

index $=$ np. linspace $(1$, len $(\operatorname{variable1})$, len(variable 1$))$

fig, axis $=$ plt.subplots $(2,2)$

axis $[0,0] \cdot p l o t$ (index, dist_eucl, 'o')

axis $[0,1] \cdot \operatorname{plot}($ index, dist_cos, 'o')

axis $[1,0] \cdot p l o t$ (index, dist_man, 'o' )

axis $[1,1] \cdot$ plot (index, dist_mah, 'og')

axis $[0,0]$. set_title ('Euclidea')

axis $[0,1]$. set 0 title ('Coseno')

axis $[1,0]$. set_title ('Manhattan')

axis [1, 1]. set_title ('Mahalanobis')

for $i$ in range (2):

for $\mathrm{j}$ in range (2):

if $\mathrm{j}==0$ :

axis $[i, j]$. set_ylabel ('Distancia')

if $\mathrm{i}==1$ : 


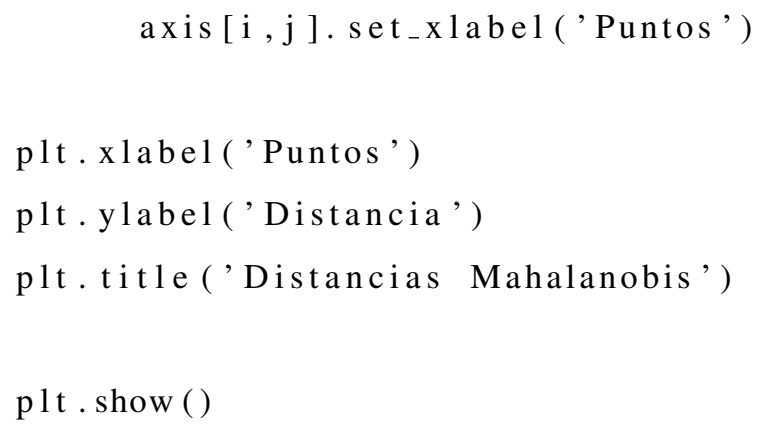

\section{Plots distancias Mahalanobis}

fig, axs $=$ plt.subplots $(1,3)$

axs [0].plot (index, dist_mah, 'o')

axs [1].plot (index, dist_covmed, 'or')

axs [2]. plot (index, dist_med, 'om')

axs [0]. axhline (y=criterio, color='k', linestyle='--', linewidth='2')

axs [1]. axhline (y=criterio, color='k', linestyle='--, , linewidth='2')

axs [2]. axhline ( $y=$ criterio, color='k', linestyle='--, , linewidth='2')

axs [0]. set_title ('Mahalanobis')

axs [1]. set_title ('Cov Mediana')

axs [2]. set_title ('Mahalanobis mediana')

\section{Scatter plots de los outliers}

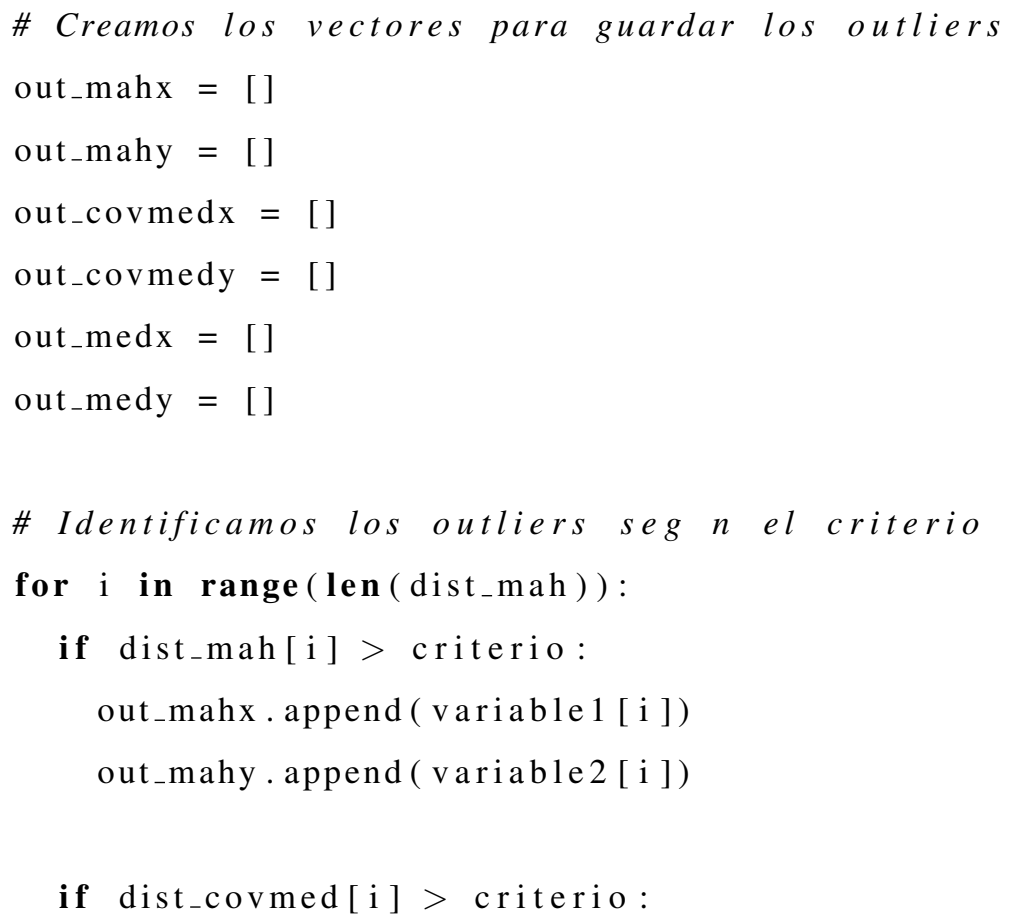




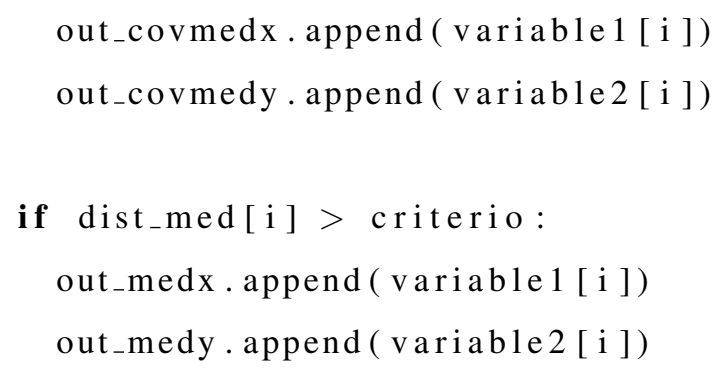

fig, axs $=$ plt. subplots $(1,3)$

axs [0].plot (variable1, variable2, 'o', label='No Outlier' )

axs[0].plot (out_mahx, out_mahy, 'or', label=' Outliers')

axs [0]. set_xlabel ('variable1')

axs [0]. set_ylabel ('variable2')

axs[0].set_title ('Mahalanobis (\%d outliers)' \% len(out_mahx))

axs $[0]$. legend ()

axs [1].plot (variable1, variable2, 'o', label='No Outlier')

axs [1].plot (out_covmedx, out_covmedy, 'or', label='Outliers')

axs [1]. set_xlabel ('variable1')

axs [1]. set_ylabel ('variable2')

axs [1]. set_title ('MAD (\%d outliers)' \% len(out_covmedx))

axs [1]. legend ()

axs [2].plot (variable1, variable2, 'o', label='No Outlier')

axs [2].plot (out_medx, out_medy, 'or', label='Outliers', )

axs [2]. set_xlabel ('variable1')

axs [2]. set_ylabel ('variable2')

axs [2]. set_title ('Mahalanobis mediana (\%d outliers)' \%len(out_medx))

axs [2]. legend ()

plt. show ()

print (len (out_mahx $)$ )

\subsection{Análisis Cluster}

\section{K-Means Clustering}




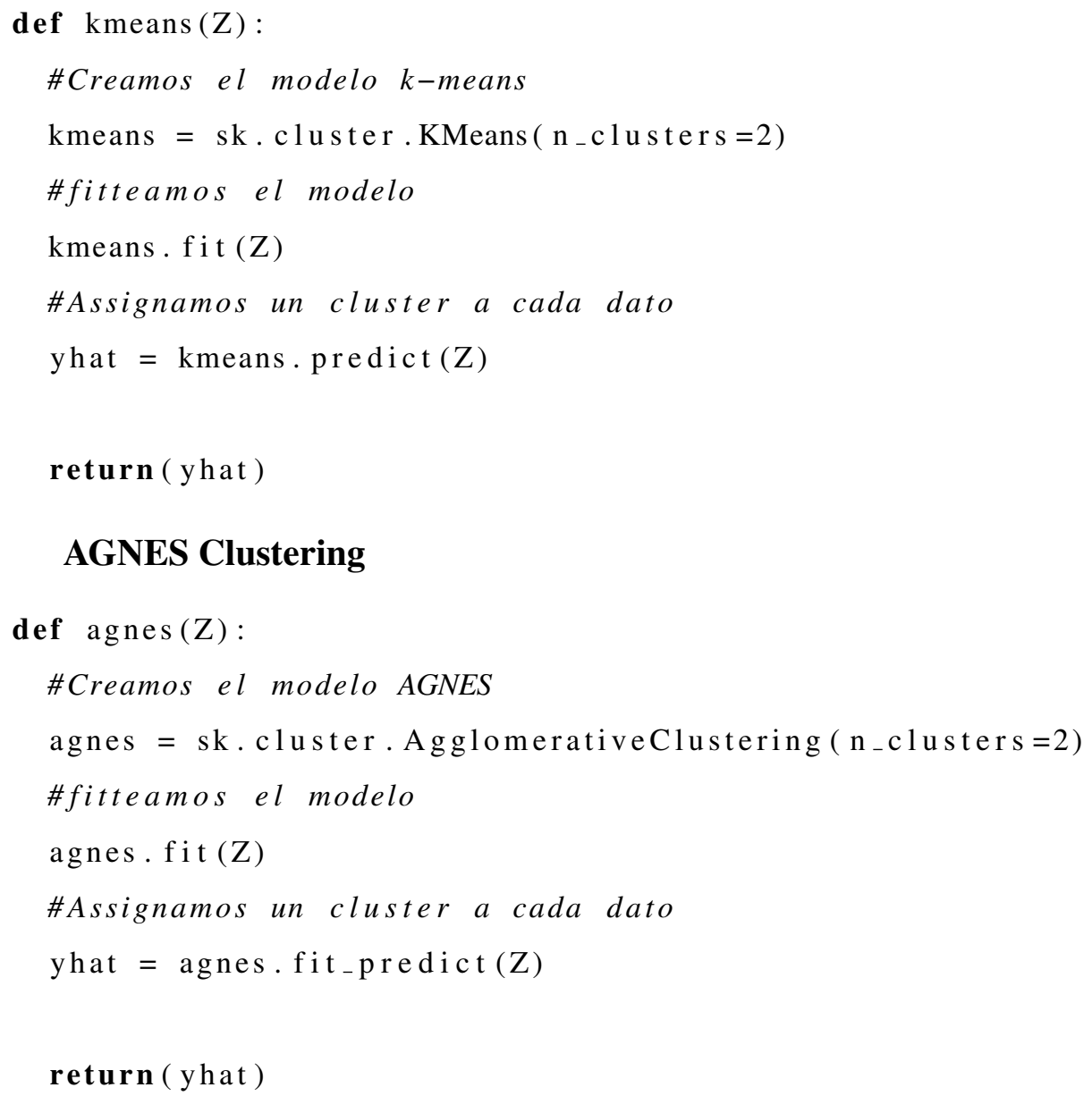

\section{Scatter Plot de los Clusters}

$Z=n p \cdot$ column_stack ( (variable 1, variable 2$))$

fig, $\operatorname{axs}=$ plt.subplots $(1,2)$

for $i$ in range (2):

if $\mathrm{i}==0$ :

yhat $=$ kmeans $(Z)$

else :

yhat $=\operatorname{agnes}(Z)$

clusters $=$ np. unique (yhat)

for cluster in clusters:

row_ix $=n p$. where $(y h a t==$ cluster $)$

axs [i].scatter $\left.\left(Z\left[\operatorname{row}_{-} i x, 0\right], Z_{[} \operatorname{row}_{-} i x, 1\right]\right)$

axs [0]. set_xlabel('variable1') 


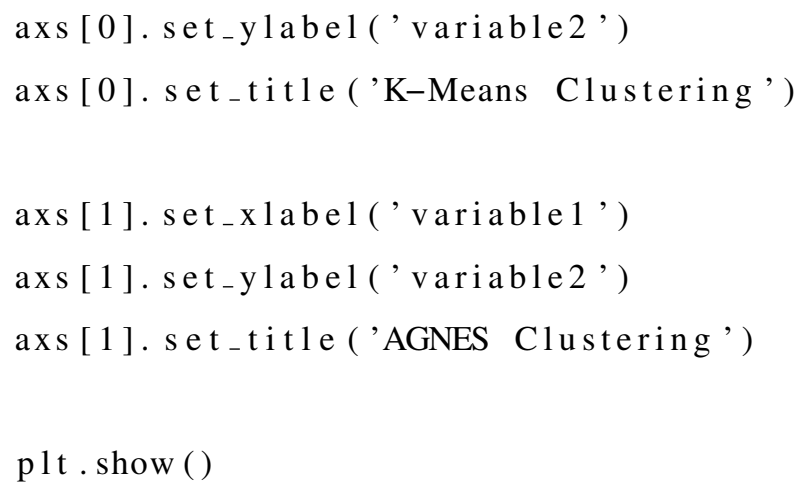

\section{Resultados}

Para el presente documento se tomaron dos bases de datos con 1259 observaciones cada una (correspondientes a un día entre el 2 de diciembre de 2015 y el 1 de diciembre de 2020). Las bases de datos contienen información de las acciones de Apple y Tesla respectivamente, como se muestra en la figura 1. Tomado de:

$$
\begin{aligned}
& \text { https://finance.yahoo.com/quote/AAPL?p=AAPL.tsrc=fin-srch } \\
& \text { https://finance.yahoo.com/quote/TSLA?p=TSLA.tsrc=fin-srch }
\end{aligned}
$$

$\begin{array}{rrrrrrrr} & \text { Date } & \text { Open } & \text { High } & \text { Low } & \text { Close } & \text { Adj Close } & \text { Volume } \\ 0 & 2015-12-02 & 29.334999 & 29.5275 & 29.020000 & 29.070000 & 26.713804 & 133546400 \\ 1 & 2015-12-03 & 29.137501 & 29.1975 & 28.555000 & 28.799999 & 26.465693 & 166278000 \\ 2 & 2015-12-04 & 28.822500 & 29.8125 & 28.777500 & 29.757500 & 27.345581 & 231108000 \\ 3 & 2015-12-07 & 29.745001 & 29.9650 & 29.452499 & 29.570000 & 27.173285 & 128336800 \\ 4 & 2015-12-08 & 29.379999 & 29.6500 & 29.215000 & 29.557501 & 27.161795 & 137238000\end{array}$

Figura 1: Datos originales

Partiendo de la idea de que el precio de cierre de la acción $x$ en el día $n$ se representa mediante la variable 'Close', se decidió tomar esta como la variable de interés a lo largo del estudio. 


\begin{tabular}{|c|c|}
\hline [ [ 29.07 & 46.397999] \\
\hline [28.799999 & 46.542 \\
\hline$[29.7575$ & 46.076 \\
\hline$[29.57$ & 46.226002 \\
\hline$[29.557501$ & 45.344002 \\
\hline [28.905001 & 44.903999 \\
\hline$[29.0425$ & 45.414001 \\
\hline [28.295 & 43.403999 \\
\hline$[28.120001$ & 43.716 \\
\hline$[27.622499$ & 44.217 \\
\hline
\end{tabular}

Figura 2: Columnas de interés para ambos activos

En la figura 3 podemos observar gráficamente el precio de las acciones para cada día, sobre el eje $\mathrm{X}$ se grafica el precio de la acción de Apple y sobre el eje Y, el de Tesla. Podemos observar, debido a la forma del Scatter Plot, que las variables están correlacionadas positivamente, es decir, si el precio de una acción aumenta, el de la otra también lo hará y viceverza.

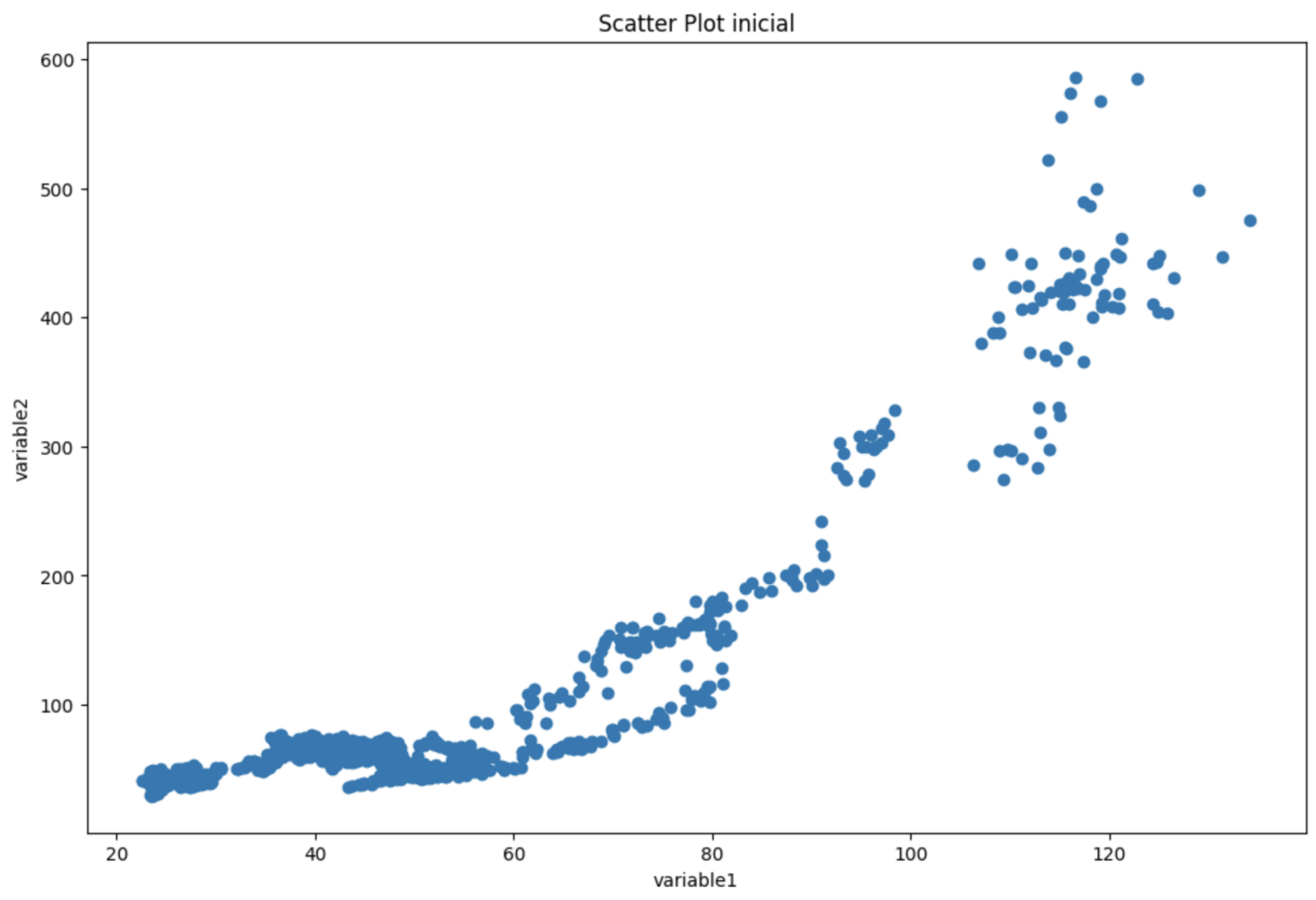

Figura 3: Scatter Plot APPL close price vs TSLA close price

Además, podemos observar a simple vista que hay dos grupos que difieren significativamente, ver figura 4. 


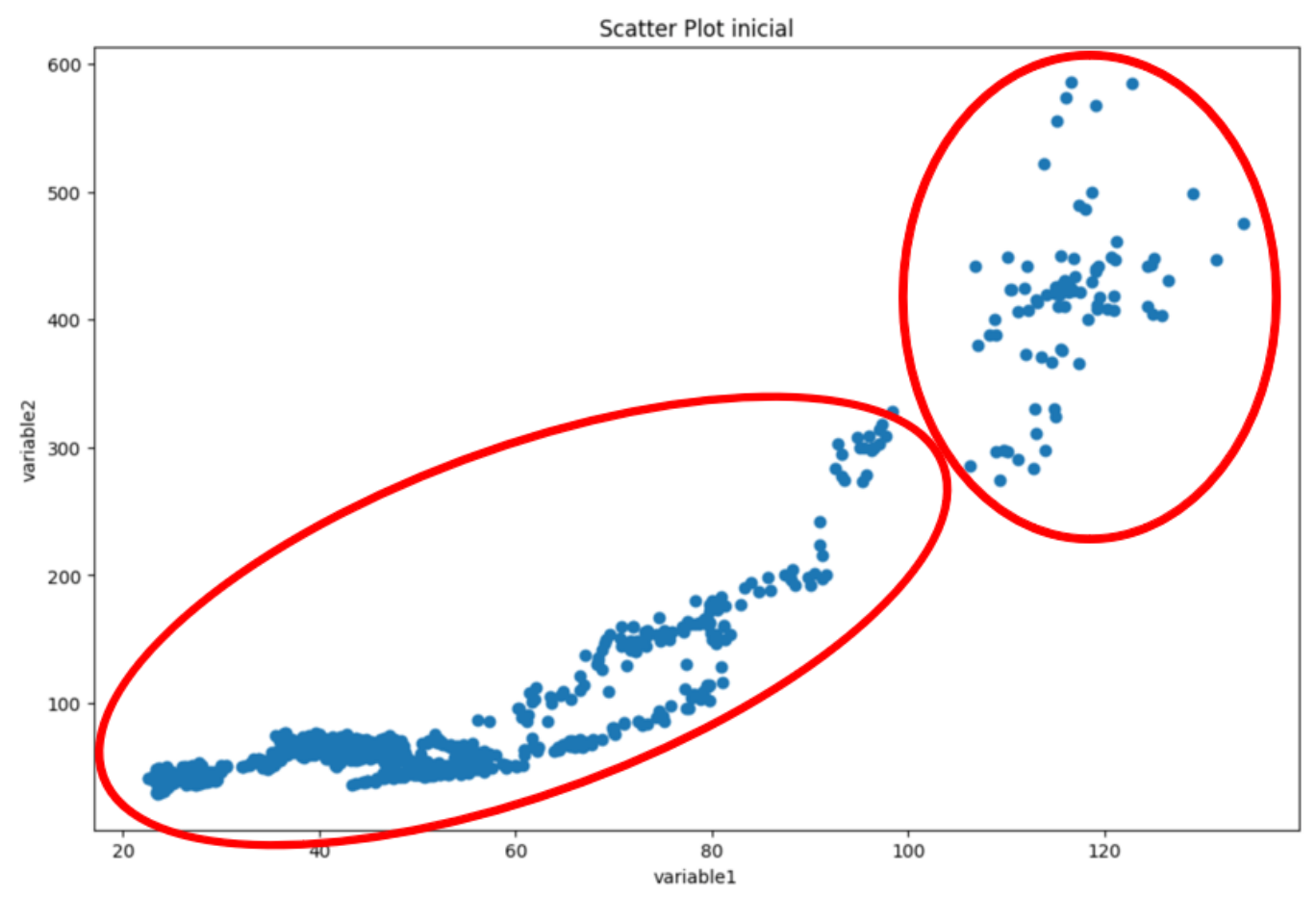

Figura 4: Grupos a priori

\subsection{Detección de Outliers mediante las métricas}

En primer lugar se calcularon las distancias de cada dato respecto a la media utilizando diferentes métricas (distancia Euclidea, distancia del Coseno, distancia de Manhattan y distancia de Mahalanobis) para posteriormente establecer un criterio mediante el cual se puedan clasificar los datos como outliers. Con base a esto, se decide solamente tomar en cuenta la distancia de Mahalanobis ya que, a diferencia de las otras métricas, esta posee un criterio de clasificación estadísticamente fundamentado el cual establece que un dato es atípico si su distancia respecto a la media es mayor al cuantil 95, matemáticamente, esto es

$$
M^{2}(i, j) \geq \chi_{0,95, n}^{2}
$$

donde $n$ son los grados de libertad (número de variables). 

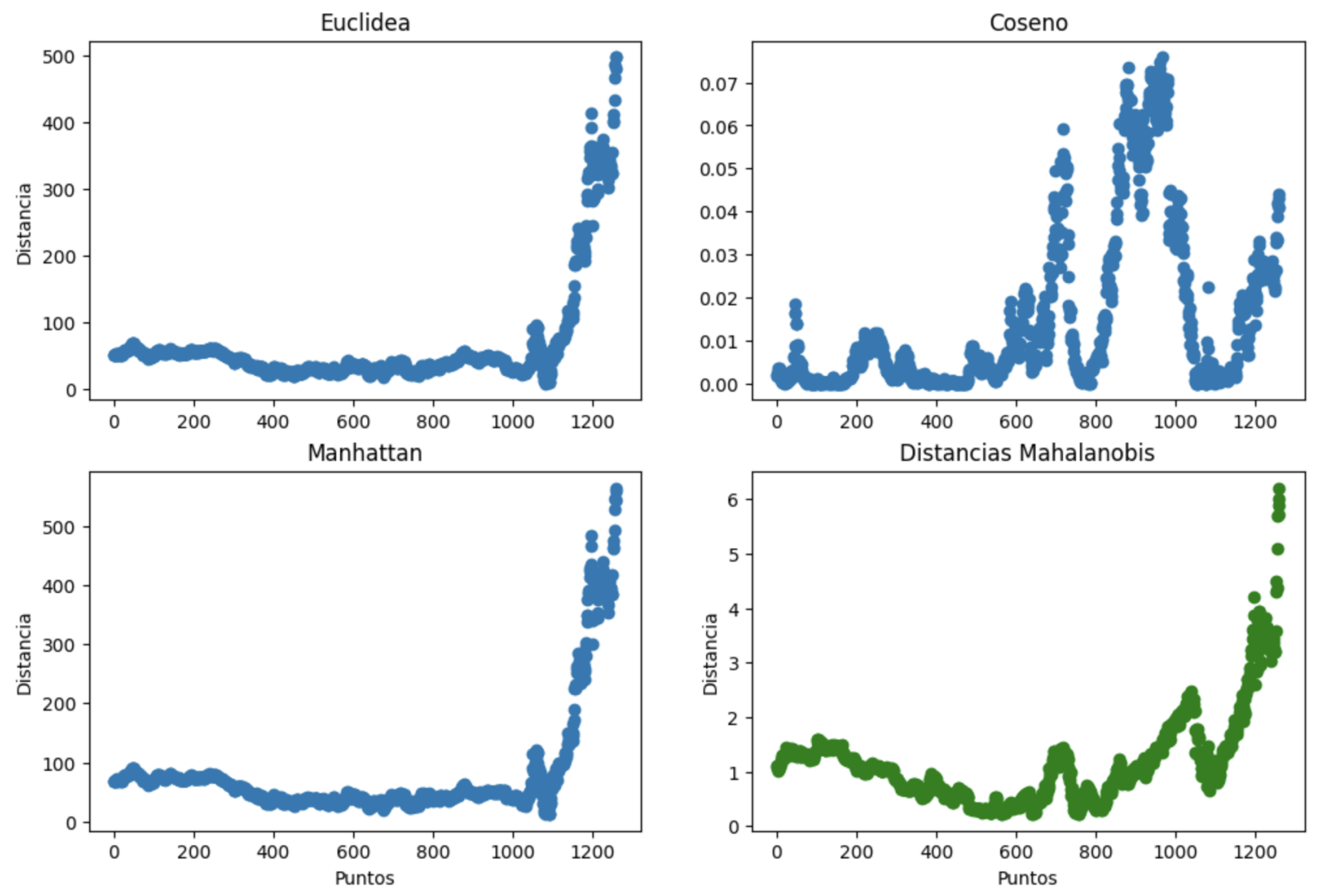

Figura 5: Distancias calculadas con los diferentes métodos

Teniendo en cuenta que la media es un estadístico sensible a datos atípicos extremos, es decir, su valor se ve afectado ante presencia de datos atípicos de gran magnitud, se propone un cálculo más robusto de las distancias y matriz de covarianzas a través de la mediana, debido a que es un estadístico menos sensible a los datos atípicos extremos y esto brindaría mayor robustez y confiabilidad a la hora de clasificar outliers [4].

En la figura 6, se muestran las tres medidas utilizadas para la identificación de outliers, donde el eje $y$ representa la distancia y el eje $x$ es cada uno de los individuos. El gráfico 'Mahalanobis' representa el cálculo clásico de la distancia de Mahalanobis; el gráfico 'Cov Mediana' representa la distancia de Mahalanobis estimada a partir de la distancia del vector respecto a la mediana y la matriz de covarianzas calculada a partir de la mediana en lugar de la media. Por último, en el gráfico 'Mahalanobis mediana' se presenta el calculo de la distancia de Mahalanobis con la modificación de calcular la distancia del vector respecto a la mediana. La línea punteada de cada gráfico representa el criterio de clasificación de datos atípicos, los puntos que se encuentren por encima de esta serán tomados como outliers. 


$$
\begin{gathered}
\text { Mahalanobis }=\left(x_{i}-\mu\right)^{\prime} \Sigma_{\text {media }}^{-1}\left(x_{i}-\mu\right) \\
\text { CovMediana }=\left(x_{i}-\text { mediana }\right)^{\prime} \Sigma_{\text {mediana }}^{-1}\left(x_{i}-\text { mediana }\right) \\
\text { MahalanobisMediana }=\left(x_{i}-\text { mediana }\right)^{\prime} \Sigma_{\text {media }}^{-1}\left(x_{i}-\text { mediana }\right)
\end{gathered}
$$

donde $x_{i}$ es un vector con los dos valores correspondientes al precio de los activos en la fecha $i$ y $\mu$ es un vector que contiene las medias correspondiente a cada activo.
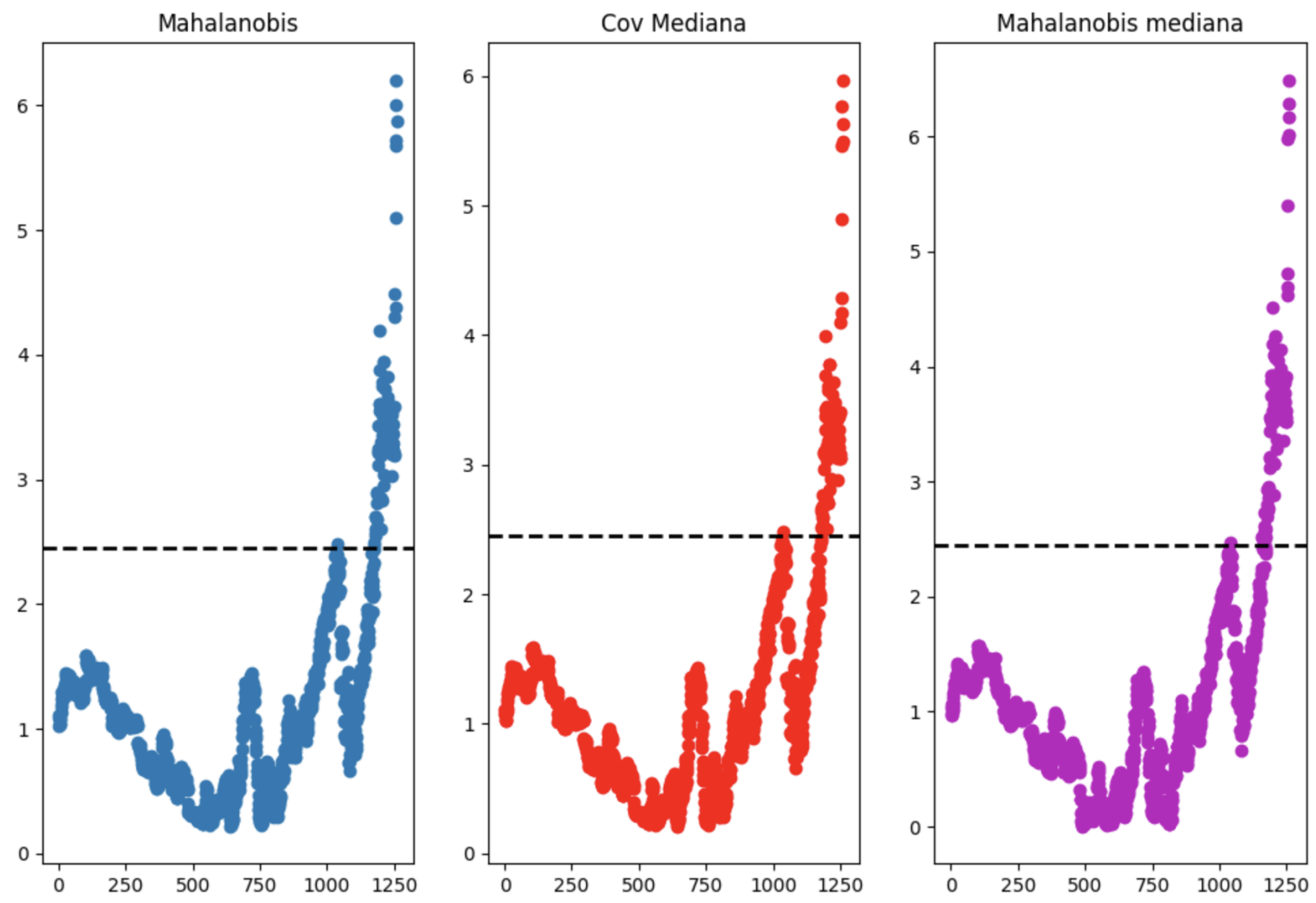

Figura 6: Distancias Mahalanobis robustas

La figura 7 presenta en color rojo los datos atípicos (outliers) clasificados por cada uno de los métodos anteriormente descritos, podemos ver que el método que más outliers identifica es el tercero, por lo que podemos concluir que tiene un mejor desempeño. 

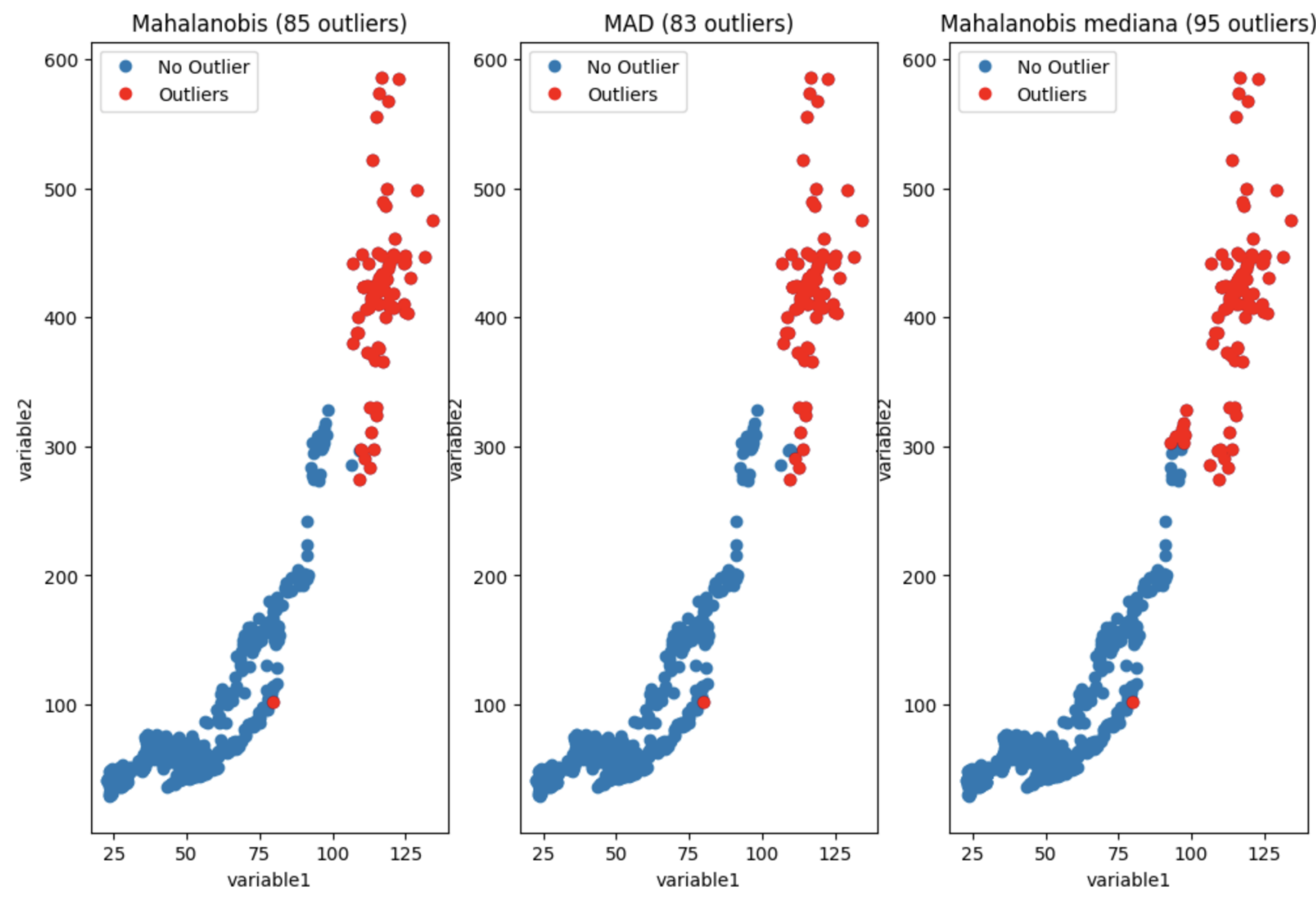

Figura 7: Identificacion de Outliers

\subsection{Análisis Cluster}

Luego de identificar los datos atípicos, se procedió a realizar el análisis cluster, para el cual se decidió hacer la agrupación en 3 grupos diferentes, pues es con el cual se presenta una mejor diferenciación de los outliers como se puede evidenciar en la figura 8, en la cual, los datos de color naranja para kmeans y color azul para AGNES, son muy similares a los datos atípicos identificados anteriormente en la figura 7. En cuanto a las dos técnicas utilizadas para la agrupación de los datos, se puede decir que no presentan diferencias significativas, sin embargo, no son iguales, pues algunos individuos pertenecen a grupos diferentes según la técnica. 

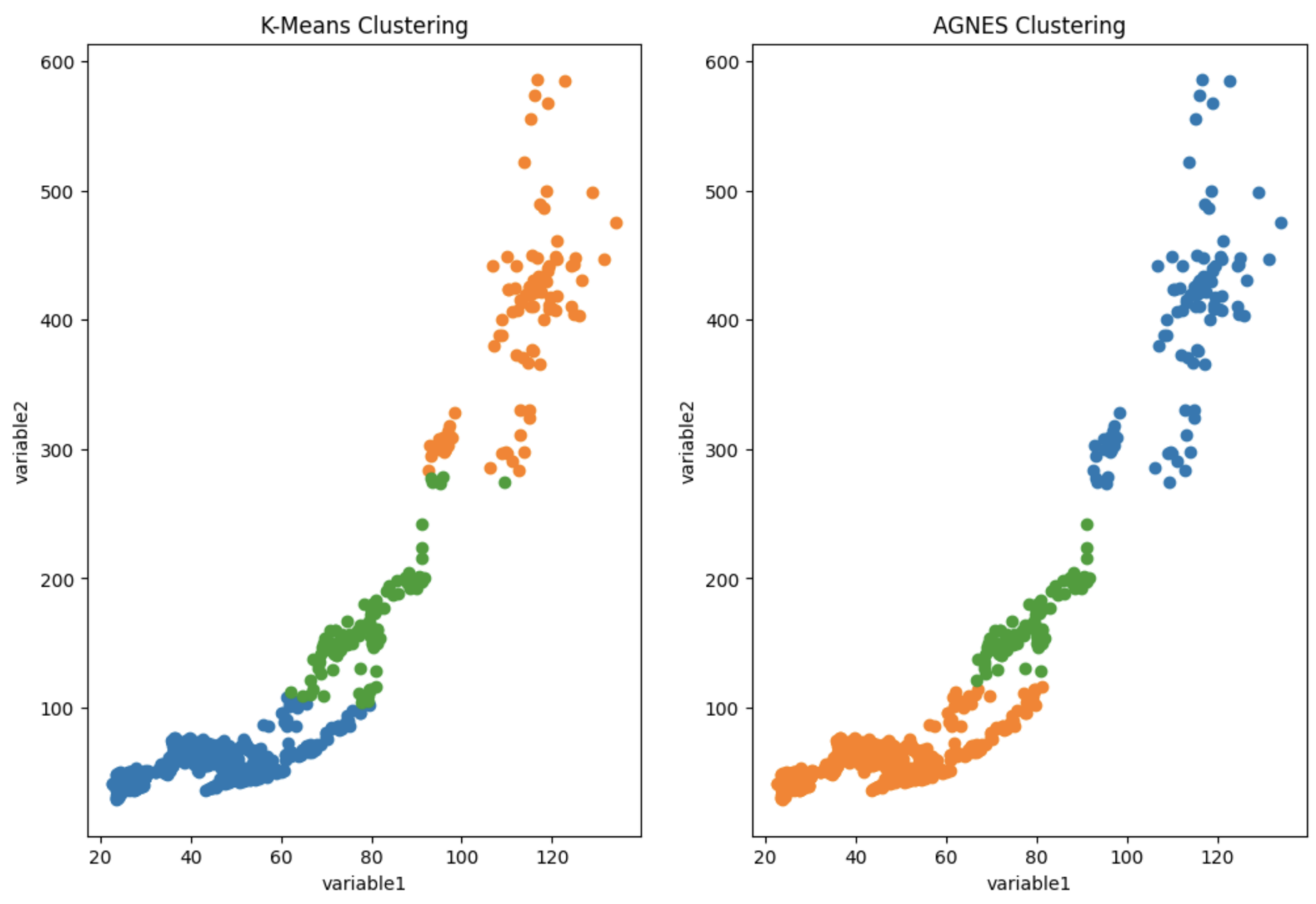

Figura 8: Scatter de los metodos clusters

\section{Conclusiones}

La elección de métricas según el objetivo y el tipo de datos que se tiene, es de gran importancia para realizar un análisis de similitud apropiado. En los enfoques estadísticos a menudo son utilizadas la distancia de Mahalanobis y la Euclidiana, mientras que para aquellos casos en los que la magnitud puede sesgar el análisis se utiliza la similitud del coseno.

La distancia de Mahalanobis da una mejor clasificación de datos atípicos debido a que existen criterios estadísticos que permiten llegar a resultados más precisos.

A partir del análisis cluster podemos concluir que los resultados obtenidos en la sección de clasificación de outliers son verídicos ya que, como podemos ver en la figura 8, el grupo naranja (para la primera imagen) y el grupo azul (para la segunda imagen) corresponden a los outliers, lo que nos quiere decir que verdaderamente dichos datos difieren significativamente del resto. 
Los precios elevados de las acciones son valores atípicos, es común que estas se mantengan en un rango entre 25 USD y 100 USD para las acciones de Apple y entre 20 USD y 300 USD para las de Tesla, aproximadamente. Además podemos observar 3 grupos en el análisis cluster, los cuales pueden ser denominados como precios bajos, medios y altos para cada acción.

\section{Referencias}

[1] A.A. Salman A. Engelbrecht M. Omran. "An overview of clustering methods". En: Department of Computer Science, School of Information Technology (2007).

[2] A. Agarwall C. Hinneburg y D. Keim. "On the Surprising Behavior of Distance Metricsin High Dimensional Space". En: Institute of Computer Science, University of Halle (2001).

[3] CARLES M. CUADRAS. "Distancias estadísticas”. En: Universitat de Barcelona (1989).

[4] Henry Laniado Santiago Medina-Hurtado Daniela Gutiérrez-Sepúlveda. "Robust estimation of the covariance matrix for the optimal selection of investment portfolios". En: Universidad Nacional de Colombia (2018).

[5] D. M. Hawkins. "Identification of Outliers". En: Chapman and Hall,London (1980).

[6] I. Kononenko y M Kukar. "Cluster Analysis. Machine Learning and Data Mining". En: (2007), págs. 321-358.

[7] B. Li y L Han. "Distance Weighted Cosine Similarity Measure for Text Classification". En: Data Mining (2013), págs. 611-618.

[8] Jorge A. Salas Plata María Teresa Escobedo Portillo. "MAHALANOBIS Y LAS APLICACIONES DE SU DISTANCIA ESTADISTICA”. En: Culcyt (2008). 